prerogative wisely in both cases and should guard against undue censorship.

Dattatray Parasnis

Gnejsstigen 6, 97753 Luleå, Sweden

Sir - Vani Kalyanaraman objects to nudity in advertisements for scientific products, but I believe that nakedness might be more appropriate here than Kalyanaraman realizes (Nature 402, 118; 1999). Given that we are being increasingly encouraged to buy almost everything via the web, perhaps use of nudity reminds us of the strong association between images of nakedness and e-commerce?

Alex May

Division of Mathematical Biology, National Institute for Medical Research, The Ridgeway, Mill Hill,

London NW7 1AA, UK

\section{EPA error risked halving India's rice harvest}

Sir - Methane has attracted particular attention as a greenhouse gas, as once it is formed, mitigation strategies are not easy to devise. The US Environmental Protection Agency (EPA) estimated in 1990 that global methane emission is about $550 \mathrm{Tg}$ (550 million tonnes) per annum, with about $110 \mathrm{Tg}$ of this released from paddy cultivation. This estimate turned out to be wrong, but it could have had devastating consequences for the economy of India.

Nearly $90 \%$ of the world's rice production comes from Asia, and rice is the staple food for 2 billion people. India produces about 80 million tonnes from about 42 million hectares. The EPA estimated methane emission from India as $37.8 \mathrm{Tg}$ per annum in $1990^{1}$. The implications of this estimate were so serious that India's National Physical Laboratory led an international collaboration of 15 laboratories to measure methane emissions directly ${ }^{2}$.

Methane released during paddy cultivation was collected and analysed, with careful controls. Methane fluxes were typically between -10 and +80 mg per hour per $\mathrm{m}^{2}$. Samples were taken from 34 sites in India of varying agricultural conditions, and measurements were done at different cultivation stages, from seedlings to harvesting, and integrated over a full year to cover single and multiple crops. Methane emission depends on the paddy-water ecosystem, and this was also measured in various ways over time.

All this direct agricultural information was used to calculate the methane emission budget $^{2}$, which was $4.07 \pm 1.25$ Tg per year, only one-tenth of the 1990 EPA estimate. A repetition of the measurements in 1996 gave essentially the same result.

The main reason for the difference between the EPA estimates and the actual measurements is that long incubation periods lead to anaerobic conditions, favouring high methane genesis. The EPA extrapolated the 1990 data without realizing that the high methane flux conditions had resulted in an annual methane emission from India that was an order of magnitude larger than the experimentally measured values.

This error has now been corrected and the estimate of global methane emission from paddy fields has been scaled down to 60 million tonnes. Methane emission from rice cultivation in India and other similar Asian countries is no longer considered a major factor in global warming.

If this error had gone uncorrected, international protocols would have required Indian methane emissions to be brought down to global average levels, reducing paddy cultivation by at least $47 \%$ in the short term. The economic cost of this would have been about 135,000 million rupees (US\$3.1 billion) per annum, nearly double the annual budget of all India's science and technology ministries combined ${ }^{3}$.

So the investments that enabled the National Physical Laboratory to perform and coordinate precise measurements saved the country from an economic crisis. This story illustrates that, even in developing countries, it is wise for governments to invest in research and development.

\section{E. S. R. Gopal}

Department of Physics, Indian Institute of Science, Bangalore 560 012, India

1. Ahuja, D. R. Estimating Regional Anthropogenic Emissions of Greenhouse Gases (US EPA Technical Series, Washington DC, 1990). 2. Parashar, D. C. et al. Chemosphere 33, 737-757 (1996).

3. Gopal, E. S. R. et al. in Proc. 2nd Int. Conf. Metrology, Quality and Global Trade (eds Banerjee, P. et al.) 432-439 (Alpha, New Delhi, 1999).

\section{Wherever HIV originated, polio vaccine is safe now}

Sir - John Moore's review of E. Hooper's The River (Nature 401, 325-6; 1999), on the possible link between the origin of HIV and a contaminated polio vaccine used during a mass vaccination campaign in equatorial Africa in the 1950s, carefully discussed the weakness of the book's hypothesis. But it is important to add that the polio vaccine currently being used worldwide is safe.

This message was not very clear when newspapers in Uganda first picked up the HIV-polio theory from the US media some years ago. As a result, some people thought that their child would be given a contaminated vaccine. So they kept their children from being vaccinated, putting them at high risk of poliomyelitis. In 1999, there were 49 confirmed polio cases in Uganda, according to the World Health Organization.

With the recent renewal of interest in the HIV-polio theory, there is a need to stress the safety of the present polio vaccine whenever it is discussed. Hopes for global eradication of polio depend largely on vaccination coverage in all parts of the world. Ka-Wing Wong

Department of Molecular Biology \& Microbiology, Tufts University School of Medicine, 136 Harrison Avenue, Boston, Massachusetts 02111, USA

\section{East German academics faced unfair hurdles}

Sir - We have some doubts about statements attributed to Jens Reich in a recent Briefing (Nature 401, 637; 1999), that some evaluation interviews conducted by the Wissenschaftsrat in Germany were held in English. Although some evaluation committees may have interviewed an East German lecturer in English or may have required someone to give a lecture in English, the claim that interviews were conducted in English must be due to a misunderstanding.

East Germany's academics were not capable of conversing in English at this level. Real proficiency in English would have aroused suspicion - either of a rightist (non-socialist) leaning or, even worse, of collaborating with the West. How could scholars or scientists have learnt a foreign language in a country such as East Germany, which disadvantaged classical and humanist studies and allowed the modern language teaching of Russian to dominate?

Of course, a knowledge of English in the former East Germany was not unheard of. A few academics who travelled abroad endeavoured to learn the language. At the end of the GDR period, language training centres were established to provide quality teaching in Russian, English, French, Spanish and Portuguese, following the example of UK language schools. These centres gave academic and other specialist staff chosen for foreign assignments an opportunity to learn their target language.

The Briefing may have overlooked an important angle. Immediately after the fall of the Berlin Wall, many heads of institutions and departments in their fifties were replaced by members of the 'pretender' generation biting the hand that had once fed them. If reunification hadn't happened, these 'pretenders' would only now be starting to reach those positions - but they would have been properly prepared by their mentors.

So, while we needed people who would stand by their principles, it was once again the Fouché-like opportunists whobelieving a political fault is worse than a crime - left the field as victors of the game. Bernd Legler

Erfenschlager Str. 150, D-09125 Chemnitz, Germany Guy Moore

Hauptstr. 73, D-53619 Rheinbreitbach, Germany 